\title{
Numerical verification of light confinement in a nano solid immersion lens
}

\author{
Myun-Sik Kim, ${ }^{1, *}$ David Nguyen, ${ }^{2}$ Alberto C. Assafrao, ${ }^{3}$ Ethan Keeler, ${ }^{4}$ \\ Skyler Rydberg, ${ }^{4}$ Gaël Osowiecki, ${ }^{2}$ Silvania F. Pereira, ${ }^{3}$ H. Paul Urbach, ${ }^{3}$ \\ Toralf Scharf, ${ }^{2}$ Hans Peter Herzig, ${ }^{2}$ Reinhard Voelkel, ${ }^{1}$ and Wataru Nakagawa ${ }^{4}$ \\ ${ }^{1}$ SUSS MicroOptics SA, Rouges-Terres 61, Hauterive, CH 2068, Switzerland \\ ${ }^{2}$ Optics \& Photonics Technology Laboratory, Ecole Polytechnique Fédérale de Lausanne (EPFL), Breguet 2, 2000 Neuchâtel, \\ Switzerland \\ ${ }^{3}$ Optics Research Group, Delft University of Technology, Lorentzweg 1, 2628 CJ Delft, The Netherlands \\ ${ }^{4}$ Dept. of Electrical and Computer Engineering, Montana State University, P.O. Box 173780, Bozeman, MT 59717-3780, USA \\ *Corresponding author: kim@suss.ch
}

\begin{abstract}
Numerical simulations using the finite element method (FEM) verify the light confinement effect observed in nano-scale solid immersion lenses (SILs). As observed in experiments, a strong light confinement occurs on the bottom surface of the SILs in simulation. The numerical spot size is comparable to the experimental spot size, and provides some insight into the deviation from the analytical expectation of the spot size reduction.

OCIS Codes: (310.6628) Subwavelength structures, nanostructures; (220.4241) Nanostructure fabrication; (260.0260)

Physical optics
\end{abstract}

\section{Introduction}

Since Mansfield invented a new immersion concept, termed the solid immersion lens (SIL) [1], numerous studies of SILs and their applications in, for example, microscopy, optical data storage and lithography, have been carried out. Initially, macroscopicsize SILs were the main subject due to the state of fabrication technologies. Recent advances in micro- and nano-fabrication technologies led to the development of different types of SILs, including diffractive SILs [2], micrometer-size SILs [3] and wavelength-scale SILs [4].

Recent experimental work has shown that subwavelength-scale SILs demonstrate a reduced-size focal spot [4]. Further investigation of nano-SILs of different shapes was reported as well [5]. However, the spot size reduction in those studies was weaker than predicted by macroscopic theory, where the refractive index $n$ of the SIL is the expected reduction factor. Since a UV polymer ( $n \sim 1.5$ at visible wavelengths) was used for the replication of the SILs, a spot size reduction of 1.5 was expected, but experimental results showed approximately 1.3 times smaller spots. Using rigorous electromagnetic simulations, we find that this reduction factor is to be expected with this size nano-SIL, indicating good agreement between the experimental measurements and the theoretical prediction.

\section{Experiments and simulations}

The fabrication process of the nano-SIL consists of three main steps. The first is electron-beam lithography (EBL) for nanopattering and structuring of the base pillars. Second, thermal reflow converts the nano-pillars into spherical caps. The EBL process is usually optimized to work with a silicon ( $\mathrm{Si}$ ) substrate that enhances the resolution by avoiding electric charging caused by non-conductive substrates. For optical characterization in transmission, such nano-structures fabricated on a $\mathrm{Si}$ substrate must be replicated on a substrate that is transparent in the visible spectrum. Thus, the final step is the replication process by soft lithography with NOA 65 UV polymer $(n=1.52)$. Fabricated nano-SILs are characterized by measuring the 3D intensity

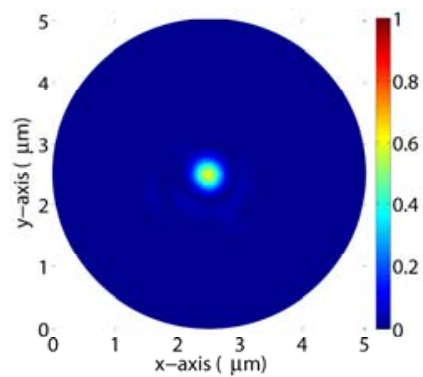

(a)

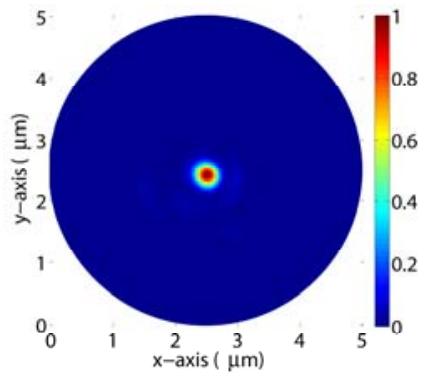

(b)

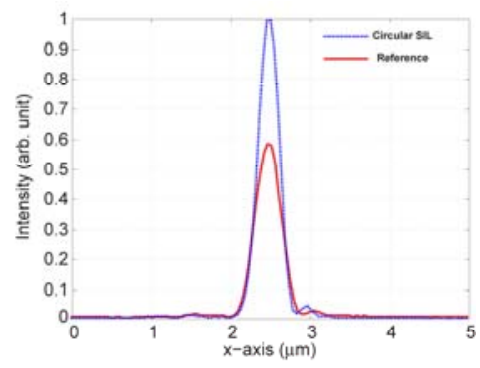

(c)

Fig. 1. Measured intensity distributions in the $x-y$ plane: (a) illumination spot (the reference non-immersion spot), (b) immersed focal spot by the nano-SIL and (c) the $x$-axis profiles of both spots. The image size is $5 \times 5 \mu \mathrm{m}^{2}$, and the intensities are normalized to the peak intensity of the immersion spot. 
distribution of the confined spot at the bottom of the SIL. A high-resolution interference microscope (HRIM), equipped with $100 \mathrm{X} / \mathrm{NA}=1.4$ oil immersion objective, is applied for such 3D intensity measurements. To focus the incident plane wave $(\lambda=$ $642 \mathrm{~nm}$ ) on the SIL, an objective of NA= 0.9 is employed.

The small size of the nano-SIL introduces some concerns when one tries to rigorously simulate the focused spot generated by these structures. Unlike the models used to describe a SIL, which usually consider an upper half space filled with the refractive index of the SIL material, the wavelength-scale dimensions of the nano-SILs require a complete mesh representation of the SIL structure. In a sense, the nano-SIL must be treated as a small scatterer, whose shape, size and orientation cannot be neglected. Since this scatterer is placed in the focal plane of a high numerical-aperture focusing lens, the vectorial nature of light must be taken into account. Hence, a rigorous simulation tool, based on solving Maxwell's equations and capable of handling complicated structures needs to be applied when analyzing such problems. Therefore, throughout this work, all simulations described are carried out in a rigorous 3D FEM simulation tool $[6,7]$. In order to simulate the NA=0.9 illumination beam, the Debye diffraction integral is solved using the Richard and Wolf method [8] that performs vectorial aplanatic focusing of high NA lenses.

\section{Results and discussions}

In the experiment, we measure the spot-size reduction by comparing the spot size created by the nano-SIL to that of the focused illumination beam (i.e., non-immersion spot). The non-immersion spot [see Fig. 1(a)] shows a full width at half maximum (FWHM) spot size of approximately $400 \mathrm{~nm}$. This reference focal spot is moved onto the 500-nm nano-SIL, and the resulting immersed focal spot is measured on the bottom of the SIL, as shown in Fig. 1(b), where the FWHM spot size is found to be 302 $\mathrm{nm}$. Corresponding simulation results for the immersion spot through the 500-nm hemispherical SIL are shown in Fig. 2. In the $x-z$ distribution, we can see the SIL body as a hemispherical cap and the illumination focal spot. The $x-y$ distribution is taken at $z$ $=-10 \mathrm{~nm}$, which represents the working plane of the SIL, and the FWHM spot size along the $x$-axis is measured to be $313.5 \mathrm{~nm}$. The simulation results show good agreement with the experimental results for the spot size reduction. As we can see in Figs. 2(a) and 2(b), the illumination spot is larger than the 500-nm SIL body. Therefore, there is an undesired loss of light that could lead to less effective light confinement, and consequently the spot size reduction does not attain the macroscopic analytical expectation of 1.5 times smaller (equal to the refractive index of the SIL). Using the rigorous simulations, we verify that measurement results do not contain noticeable experimental errors and clearly demonstrate the origin of the loss of the spot size reduction. In the future, we will use these tools to further optimize the design of the nano-SILs, taking into consideration both the minimum spot size and the ease of fabrication of such structures.

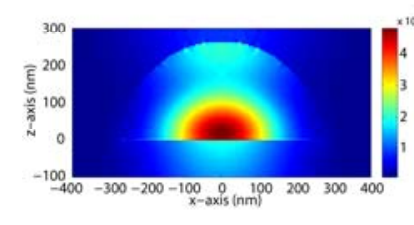

(a)

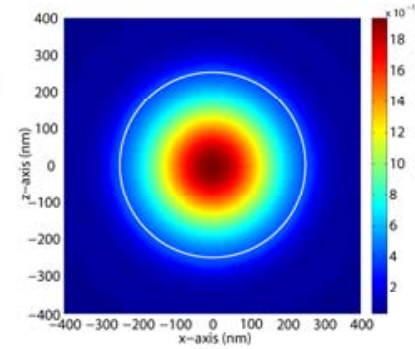

(b)

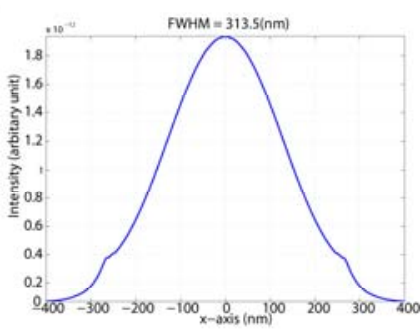

(c)

Fig. 2. Simulated intensity distribution: (a) the $x$-z plane at $y=0$, (b) and the $x-y$ plane at $z=-10 \mathrm{~nm}$, where the white circle indicates the SIL. (c) The $x$-axis profile shows the FWHM spot size of $313.5 \mathrm{~nm}$.

\section{References}

[1] S. M. Mansfield and G. S. Kino, "Solid immersion microscope," Appl. Phys. Lett. 57, 2615 (1990).

[2] R. Brunner, M. Burkhardt, A. Pesch, O. Sandfuchs, M. Ferstl, S. Hohng, and J. O. White, "Diffraction-based solid immersion lens," J. Opt. Soc. Am. A 21, 1186 (2004).

[3] D. A. Fletcher, K. B. Crozier, C. F. Quate, G. S. Kino, K. E. Goodson, D. Simanovskii, and D. V. Palanker, "Near-field infrared imaging with a microfabricated solid immersion lens," Appl. Phys. Lett. 77, 2109 (2000).

[4] M.-S. Kim, T. Scharf, M. T. Haq, W. Nakagawa, and H. P. Herzig, "Subwavelength-size solid immersion lens,” Opt. Lett. 36, 3930 (2011).

[5] M.-S. Kim, T. Scharf, D. Nguyen, E. Keeler, S. Rydberg, W. Nakagawa, G. Osowiecki, H. P. Herzig, and R. Voelkel, "Light confinement effect of nonspherical nanoscale solid immersion lenses," Proc. of SPIE Vol. 8613, 86131B (2013).

[6] A. J. Wachters and H. P. Urbach, Technical Note Phillips Research Europe, PR-TN 00042 (2008).

[7] X. Wei, A. J. Wachters, and H. P. Urbach, “Finite-element model for three-dimensional optical scattering problems," J. Opt. Soc. Am. A 24, 866 (2007).

[8] B. Richards and E. Wolf, "Electromagnetic diffraction in optical systems, II Structure of the image field in an Optical system," Proc. R. Soc. Lond. A. 253(1274), $358(1959)$.

\section{Acknowledgements}

The research leading to these results has received funding from the European FP7 Project SEAL (FISMA), the Swiss CTI-Projects NanoHyMo, AMALITH and the U.S. National Science Foundation (NSF). 